February 2000 - NREL/CP-500-27470

\title{
Wind Turbine Design Codes: A Comparison of the Structural Response
}

M.L. Buhl Jr., A.D. Wright, and K.G. Pierce

Presented at the $19^{\text {th }}$ American Society of Mechanical Engineers (ASME) Wind Energy Symposium Reno, Nevada January 10-13, 2000

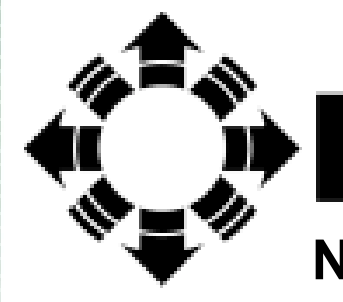

\section{NPE}

National Renewable Energy Laboratory

1617 Cole Boulevard

Golden, Colorado 80401-3393

NREL is a U.S. Department of Energy Laboratory

Operated by Midwest Research Institute $\bullet$ Battelle $\bullet$ Bechtel

Contract No. DE-AC36-99-G010337 


\section{NOTICE}

The submitted manuscript has been offered by an employee of the Midwest Research Institute (MRI), a contractor of the US Government under Contract No. DE-AC36-99G010337. Accordingly, the US Government and MRI retain a nonexclusive royalty-free license to publish or reproduce the published form of this contribution, or allow others to do so, for US Government purposes.

This report was prepared as an account of work sponsored by an agency of the United States government. Neither the United States government nor any agency thereof, nor any of their employees, makes any warranty, express or implied, or assumes any legal liability or responsibility for the accuracy, completeness, or usefulness of any information, apparatus, product, or process disclosed, or represents that its use would not infringe privately owned rights. Reference herein to any specific commercial product, process, or service by trade name, trademark, manufacturer, or otherwise does not necessarily constitute or imply its endorsement, recommendation, or favoring by the United States government or any agency thereof. The views and opinions of authors expressed herein do not necessarily state or reflect those of the United States government or any agency thereof.

Available electronically at http://www.doe.gov/bridge

Available for a processing fee to U.S. Department of Energy and its contractors, in paper, from:

U.S. Department of Energy

Office of Scientific and Technical Information

P.O. Box 62

Oak Ridge, TN 37831-0062

phone: 865.576 .8401

fax: 865.576.5728

email: reports@adonis.osti.gov

Available for sale to the public, in paper, from:

U.S. Department of Commerce

National Technical Information Service

5285 Port Royal Road

Springfield, VA 22161

phone: 800.553 .6847

fax: 703.605.6900

email: orders@ntis.fedworld.gov

online ordering: http://www.ntis.gov/ordering.htm

Printed on paper containing at least $50 \%$ wastepaper, including $20 \%$ postconsumer waste 


\title{
WIND TURBINE DESIGN CODES: A COMPARISON OF THE STRUCTURAL RESPONSE
}

\author{
Marshall L. Buhl, Jr. , Alan D. Wright, ${ }^{+}$Kirk G. Pierce ${ }^{\ddagger}$ \\ National Renewable Energy Laboratory \\ Golden, Colorado
}

\begin{abstract}
\$
The National Wind Technology Center (NWTC) of the National Renewable Energy Laboratory is continuing a comparison of several computer codes used in the design and analysis of wind turbines. The second part of this comparison determined how well the programs predict the structural response of wind turbines. In this paper, we compare the structural response for four programs: ADAMS, BLADED, FAST_AD, and YawDyn. ADAMS is a commercial, multibody-dynamics code from Mechanical Dynamics, Inc. BLADED is a commercial, performance and structural-response code from Garrad Hassan and Partners Limited. FAST_AD is a structural-response code developed by Oregon State University and the University of Utah for the NWTC. YawDyn is a structural-response code developed by the University of Utah for the NWTC. ADAMS, FAST_AD, and YawDyn use the University of Utah's AeroDyn subroutine package for calculating aerodynamic forces. Although errors were found in all the codes during this study, once they were fixed, the codes agreed surprisingly well for most of the cases and configurations we evaluated. One unresolved discrepancy between $B L A D E D$ and the AeroDyn-based codes was when there was blade and/or teeter motion in addition to a large yaw error.
\end{abstract}

\section{INTRODUCTION}

Because of the difficulty and expense of doing field tests to certify wind-turbine structures, the U.S. Department of Energy's Wind Energy Program has developed several wind-turbine design codes. Manufacturers can use these codes to predict both the extreme

\footnotetext{
* Senior Engineer I, National Wind Technology Center

† Senior Engineer II, National Wind Technology Center

* Engineer II, National Wind Technology Center

$\S$ Copyright (C) 2000 by the American Institute of Aeronautics and Astronautics, Inc. No copyright is asserted in the United States under Title 17, U.S. Code. The U.S. Government has a royalty-free license to exercise all rights under the copyright claimed herein for Governmental Purposes. All other rights are reserved by the copyright owner.
}

loads and the fatigue life of their turbines. Before the predictions from these codes will be accepted by certifying agencies, the codes must be rigorously tested. Germanischer Lloyd, a certifying agency in Germany, has accepted $B L A D E D$ as a suitable program for use in the calculation of wind turbine loads for design and certification.

One of the first steps in ensuring the quality of these predictive codes is to compare them to hand calculations and to programs that have gained general acceptance. The focus of this paper is on comparisons between codes.

A previous paper by Buhl et $\mathrm{al}^{1}$ made a preliminary comparison of the aerodynamic calculations of the codes. Since then, some of the aerodynamic algorithms have been modified, and we have repeated that comparison. The predictions of the codes are now close enough to allow comparisons of the structural loads predictions. We will not show results of the aerodynamic comparisons in this paper but will concentrate on the structural response.

In this paper, we compare the predictions of four programs: ADAMS, BLADED, ${ }^{2}$ FAST_AD, ${ }^{3}$ and YawDyn. ${ }^{4}$ ADAMS is a commercial, multibody-dynamics code from Mechanical Dynamics, Inc. $B L A D E D$ is a commercial, performance and structuralresponse code from Garrad Hassan and Partners Limited. FAST_AD is a structural-response code developed by Oregon State University and the University of Utah for the National Wind Technology Center (NWTC). YawDyn is a structural-response code developed by the University of Utah for the NWTC. ADAMS, FAST_AD, and YawDyn use the University of Utah's AeroDyn ${ }^{4}$ subroutine package for calculating aerodynamic forces.

We modeled an approximation of the AWT-27 P4 turbine with the four simulators. The AWT-27 is a twobladed, teetering, free-yaw, downwind turbine with $7^{\circ}$ of precone. We carefully used similar properties for all simulators. Because we are not comparing predictions to test data for this study, it is far more important to use the same properties for all simulators than it is to accurately model the real turbine. Although the properties 
used are not quite the same as those of the P4, they are close enough to be representative of a realistic, utilityscale wind turbine. For this study, we call the turbine we modeled the AWT-27CR (code runoff).

Before adding structural degrees of freedom (DOFs), we examined the aerodynamic loads predicted by the programs using their common aerodynamic features. Although not exactly the same, the predictions were close enough to enable us to proceed with an examination of the structural response.

Next, we operated a rigid version of the turbine in a vacuum to see if our mass properties were consistent among the programs. We then added the teeter DOF to see if the dynamic responses agreed.

After ensuring ourselves that we were using similar, rigid-body properties, we started exciting the models with various types of wind inflow. For our studies, the inflow consisted of steady winds of 6,12 , and $18 \mathrm{~m} / \mathrm{s}$, transient winds, and stochastic, full-field turbulent winds. For transient winds, we used four of the standard International Electrotechnical Commission (IEC) $61400-1^{5}$ extreme wind conditions. These were various kinds of extreme gusts and direction changes. We used SNLWIND-3D ${ }^{6}$ to generate the full-field wind files. We modified it and AeroDyn to create and use files compatible with $B L A D E D$ 's full-field wind files. This made it possible for all simulators to use the exact same inflow.

For each of the inflow cases, we used the simulators to predict the structural response. We started with a rigid turbine, then added new DOFs in steps until we were using fully flexible rotors with free teeter.

In this paper, we will list the aerodynamic features used in all the programs. We will also explain how the various programs model the turbine structure and describe the DOFs used for this study.

To compare the responses, we used NWTC-developed postprocessing programs called GPP ${ }^{7}$ and $\mathrm{Crunch}^{8}$ to compute power spectral densities (PSDs), probability densities (PDs), rainflow cycle counts, and azimuth averages. We will show some of the results in this paper.

\section{SIMULATOR CAPABILITIES}

The various simulators use somewhat different techniques to model the aerodynamics and the turbine structure. They do not all model the same aerodynamic features and have different structural DOFs. For this comparison, we tried to use features that were common to all simulators. A list of programs and the versions we used appears in Table 1.

The AeroDyn aerodynamics subroutine package, developed for the NWTC by the University of Utah, is used in three of our simulators: ADAMS, FAST_AD, and YawDyn. It was written in a modular form that allows it to be readily interfaced to structural-dynamics codes. It can read hub-height wind files with wind shear and gusts. It can also read full-field turbulence files created by SNLWIND-3D. It uses blade-element momentum theory, a Pitt and Peters' dynamic inflow model, and a Leishman-Beddoes dynamic stall model.

ADAMS is a multibody-dynamics code that we linked with AeroDyn. It is a sophisticated program that can model virtually any type of turbine. It is not actually a wind turbine code and is often used to analyze cars, robots, and spacecraft. It is a well-tested program, and we believe the rigid-body predictions of this fully nonlinear code are very accurate. ADAMS uses lumped masses connected by flexible fields similar to multidimensional spring dampers to model flexible bodies like blades and towers. This technique uses some approximations and is not as exact as the rigid-body portion of the simulator. Using a finite-element method would probably be more accurate. For our AWT_27CR, we used 11 lumped masses for each blade separated by 10 flexible field statements.

Unlike ADAMS, BLADED is a wind-turbine specific code. It is actually a suite of programs that are all initiated through an easy-to-use graphical interface. $B L A D E D$ has undergone extensive testing and is accepted by the German certifying agency, Germanischer Lloyd, as "a suitable program for the calculation of wind turbine loads for design and certification." Because it is a wind turbine specific code, it has limited DOFs but can model many common turbine configurations. Because it lacks the ability to put the teeter pin anywhere except at the point where the blades intersect the hub, we modeled the AWT-27CR this way in all simulators.

$B L A D E D$ models flexible elements using modal representation. The reliability of this representation depends on the generation of accurate mode shapes and frequencies. The blade model uses properties such as stiffness and mass per unit length to specify the flexibility characteristics. Before a response simulation can be done, users execute a modal analysis calculation to generate the mode shapes and frequencies for the rotating blades. Although the mode shapes do not change with rotational speed, their frequencies vary to account for centrifugal stiffening. For our AWT_27CR model, we specified blade properties at 27 locations along the blade.

Like AeroDyn, BLADED also uses blade-element momentum theory to model the airflow. It can also use a dynamic inflow model based upon the method of Pitt and Peters, and has a dynamic stall model based upon the work of Leishman and Beddoes. The implementa- 
tions of these algorithms are probably not the same as those in AeroDyn, so we did not use either dynamic inflow or dynamic stall in this study. We found some differences in the equilibrium-inflow algorithms, and we modified AeroDyn to use algorithms more similar to $B L A D E D$ 's.

FAST_AD has modeling features similar to $B L A D E D$. It has similar DOFs and also uses modal representation to model flexible structures. FAST_AD has its own utility program to generate mode shapes, but we used the ones generated by $B L A D E D$ to give us the best chance of fair comparisons. One shortcoming of the current version of FAST_AD is that the modal damping for higher-order modes must be the same as that for the first mode.

YawDyn is the simplest of the structural-response codes. For teetering turbines, it has only two DOFs, teeter and yaw. It models the rotor as a single rigid body, so there is no flexibility in the blades. In the data we present, we may say that the results are for flexible blades, but in the case of YawDyn, they are rigid. Like ADAMS and FAST_AD, YawDyn uses the AeroDyn routines for aerodynamics.

Because it is easier to convert from mass/length distributions to lumped masses, we derived the ADAMS properties from the distributions we used for BLADED and FAST_AD. For this, we used an Excel spreadsheet to calculate the mass and centers of mass (CMs) for the lumped-mass parts. Once the ADAMS model had been built we used the ADAMS/View aggregate-mass information feature to find the mass properties we needed for YawDyn. These values agreed extremely well with the mass totals reported by $B L A D E D$.

This process of getting mass properties to agree took much longer than expected. Part of this was caused by the lack of a thorough understanding of all the codes. We discovered many unexpected limitations during this process.

Two features in $B L A D E D$ required us to modify our rotor models. One was that $B L A D E D$ requires that the teeter axis be located at the point where the blade spars intersect the shaft. This is often not the case for teetering turbines, so we had to modify all models to agree with what we could model with $B L A D E D$. The other feature was that none of the hub mass teeters in $B L A D E D$. This forced us to include the hub mass in the blades. We do not have any stiffness data for the AWT-27 hub, so we gave that part of the rotor a stiffness that is approximately ten times higher than that for the blade root section. We modeled the rotor in FAST_AD the same as we did for BLADED.

A side benefit of including the hub as part of the flexible blades was that we could get bending loads at the center of rotation for BLADED and FAST_AD.
This did not matter for ADAMS, as we could put virtual strain gages anywhere on the turbine. We had earlier eliminated YawDyn from this study because it could only report blade loads at the center of rotation. These modifications allowed us to reintroduce YawDyn to the comparison.

Table 1. Simulators Used in This Study.

\begin{tabular}{|l|l|}
\hline Program & Version \\
\hline ADAMS & 9.1 (Patch 91-33), AeroDyn 11.21cr-e \\
\hline BLADED & 3.3 \\
\hline FAST_AD & 2.1, AeroDyn $11.21 \mathrm{cr}-\mathrm{e}$ \\
\hline YawDyn & $11.21 \mathrm{cr}-\mathrm{e}$, AeroDyn $11.21 \mathrm{cr}-\mathrm{e}$ \\
\hline
\end{tabular}

\section{AERODYNAMICS}

Because the thrust of this phase of our study was to compare the structural responses of the simulators, we attempted to use the same aerodynamic features from each of the codes. Without similar aerodynamic input, comparisons of structural responses are impossible. Although the codes can model aerodynamic effects such as dynamic wake, dynamic stall, and tower shadow, we did not use them in this phase of the study. The aerodynamic features we used were: axial induction, tangential induction, equilibrium inflow, Prandtl tip loss (no hub loss), and wind shear.

In our first paper, ${ }^{1}$ we mentioned a difference in how the codes used the tip-loss correction factor in the induction-factor calculation. This difference caused a significant disagreement in the aerodynamic loads in the outboard portion of the blade. We conferred with Robert Wilson of Oregon State University and Michael Selig of the University of Illinois at Urbana-Champaign. They both agreed that BLADED uses the correct method. We modified AeroDyn to use the correct algorithm, and now the codes agree quite well in the outboard portion of the blade.

There still seemed to be a significant difference in the inboard portions of the blades. When looking at the tangential induction factor, we noticed that the codes predicted very different values for stations where the angle of attack increased enough to produce large drag coefficients. We examined the documentation for $B L A D E D$ and found that it includes the drag term in both the axial and tangential induction-factor calculations. AeroDyn does not use the drag terms. We modified AeroDyn to include the drag terms and got a much better agreement. There are still some differences at low wind speeds, but at $18 \mathrm{~m} / \mathrm{s}$ the predictions are virtually identical. We made this modification only for this 
study, and it is not included in the released version of AeroDyn.

Although there has been some debate over the years as to whether or not these drag terms are appropriate, AeroDyn included them at one time. However, Robert Wilson's view is that because drag is not an action-at-adistance force as the lift is, drag has only a localized effect on the induced velocities and should not be used in the calculations. ${ }^{9}$

\section{STRUCTURAL DEGREES OF FREEDOM}

As we mentioned in the section describing the capabilities of the simulators, we modeled blade flexibility in ADAMS as a series of lumped masses separated by flexible fields similar to spring dampers. BLADED and FAST_AD use the method of modal representation. YawDyn cannot model blade flexibility for teetering rotors.

The only other DOF we enabled for this phase of our study was the teetering DOF. We do not, however, model teeter dampers with a typical deadband or with teeter stops. We put a light spring $(1 \mathrm{kN} \mathrm{m})$ on the teeter that engages immediately and has enough damping (40 $\mathrm{kN} \mathrm{m} \mathrm{s}$ ) to get the models to behave in a vacuum.

We tried enabling the yaw DOF in all the codes, but the responses were very dissimilar. It seemed meaningless to compare the loads from the simulators when the models yawed in different, and sometimes opposite, directions. Lacking time to determine and fix the causes of the differences, we decided to eliminate that DOF from this phase of our study. We also did not have time to enable tower or drive-train flexibility.

\section{WIND CONDITIONS}

We used several different types of inflow to excite our structural models. The first case was no inflow. Operating the turbine models in a vacuum helped us find errors in our models and in the codes. After we got good agreement for the zero air-density case, we used steady, non-sheared winds for the inflow. We then proceeded to sheared flow. For both sheared and nonsheared flow, we used wind speeds of 6,12 , and $18 \mathrm{~m} / \mathrm{s}$, which provided a reasonable range of speeds for the purposes of this study.

Once we enabled teeter and blade flexibility, and ensured that we had the best comparisons, we used the four IEC extreme-wind conditions listed in Table 2.

Next, we used full-field turbulent wind data from SNLWIND-3D to excite the structure. We ran three 10minute simulations at mean u-component wind speeds of 6,12 , and $18 \mathrm{~m} / \mathrm{s}$.
Table 2. IEC Extreme Wind Conditions.

\begin{tabular}{|l|l|}
\hline EDC_r & Extreme direction change at rated wind speed $(17 \mathrm{~m} / \mathrm{s})$ \\
\hline EDC_o & Extreme direction change at cut-out wind speed $(22.5 \mathrm{~m} / \mathrm{s})$ \\
\hline ECG & Extreme Coherent Gust \\
\hline ECD & Extreme Coherent Gust with Direction Change \\
\hline
\end{tabular}

\section{DATA ANALYSIS}

For our comparisons, we analyzed four parameters: teeter angle, blade out-of-plane (OoP) bending moment at the center of rotation, in-plane (IP) bending moment at the center of rotation, and yaw moment.

Although we started to compare blade-tip deflections, we dropped them from the analysis. Because YawDyn does not have flexible blades, and $B L A D E D$ leaves out the effects of gravity in their blade deflections, there seemed little reason to compare them. We also did not compare shaft torque or power, because we assumed they were essentially driven by the IP blade moments.

For all simulations, we eliminated the first $10 \mathrm{sec}-$ onds of the output to be sure the turbine had reached a steady state. For the steady wind cases, we used Crunch v1.74 to generate azimuth averages of the signals. For the IEC extreme wind conditions, we compared the time series. For the full-field turbulence cases, we used Crunch to generate PDs and RCCs, and GPP v6.00 to generate PSDs.

We agonized about the choice of scales for the plots. To zoom in on the curves to magnify the differences in the predictions, might tell a misleadingly pessimistic story. Instead, we chose to find the minimum and maximum of each of the signals for all test cases and use them to set our scales. We believe this tells a more meaningful story.

\section{COMPARISONS}

\section{Non-Teetering Turbine with Rigid Blades}

The first step in our structural comparisons was to set the air density to zero and disable all flexibility and teetering. This enabled us to ensure we had the same dimensional, mass, and inertial properties. We also calculated the blade loads for the horizontal and vertical configurations in a spreadsheet. At first, the codes did not agree, so we tried setting the gravity to zero to compare only the centrifugal loads. These steps helped us find errors in our models and in the simulators.

After getting excellent agreement for the models operating in a vacuum, we set about getting the blade-element, aerodynamic properties to agree. To do this, we made several modifications to AeroDyn to give us 
reasonably good agreement with $B L A D E D$. Modifications included adding the drag terms to the induction equations, using a different equation for the thrust coefficient, and disabling the skewed-wake corrections. We also set the air density to sea-level density and excited the rigid-turbine models with steady winds. We did this for both sheared and non-sheared flow at $0^{\circ}$ yaw error. The blade loads from each simulator were very close for both sheared and non-sheared winds. The yaw moments for the sheared-flow cases were exactly the same at moderate and high wind speeds, but merely close at $6 \mathrm{~m} / \mathrm{s}$. In non-sheared flow, there is no yaw moment for the non-yawed condition, so all codes predicted no yaw moment.

We also ran the same cases with $15^{\circ}$ and $30^{\circ}$ yaw errors. All the codes agreed perfectly for both sheared and non-sheared flow. See Figures 1 and 2 for examples of these loads for sheared, $12 \mathrm{~m} / \mathrm{s}$ steady winds, and a yaw error of $30^{\circ}$.

\section{Teetering Turbine With Rigid Blades}

We next turned on the teetering DOF and set the air density to zero. Plots of azimuth averages showed that the predictions of all parameters were virtually identical for all four codes, which gave us the confidence we needed to move forward.

For both sheared and non-sheared winds, we got excellent agreement for the blade loads. For teeter angle, $B L A D E D$ predicted about twice the deflection as the AeroDyn-based codes at $6 \mathrm{~m} / \mathrm{s}$. Granted, these deflections were only a fraction of a degree. At higher wind speeds, BLADED's teeter deflections were closer to those of the AeroDyn-based codes, which had differences amongst themselves. These differences in teeter deflections seem to have affected the yaw-moment predictions. For the cases with yaw errors, BLADED predicted much larger teeter angles than the AeroDynbased codes, which exhibited virtually the same response. Again, this also showed up in the yaw response. For blade loads, the codes gave excellent agreement at 6 and $12 \mathrm{~m} / \mathrm{s}$, but we found a problem in our modified version of AeroDyn at $18 \mathrm{~m} / \mathrm{s}$. The codes seemed to have a hard time converging. Because the problem does not appear in the normal version of AeroDyn, we decided it was not worth the effort to find the problem. This problem did not occur at lower wind speeds, as you can see in Figure 3, which plots the 12 $\mathrm{m} / \mathrm{s}$ data.

Because the differences seem to appear only when there is blade motion, we suspect that the differences we found for configurations with free teeter were caused by a difference in how the aerodynamic routines account for the motion of the blades. The problem seems exacerbated by high yaw errors. As of this writing, we do not know which of the two is more accurate.

\section{Non-Teetering Turbine with Flexible Blades}

We added flexibility to the blades for ADAMS, $B L A D E D$, and FAST_AD. YawDyn does not allow any blade flexibility for teetering turbines. The lack of blade flexibility for YawDyn is evident from the plots of blade bending moments, as shown in Figure 4. Because the YawDyn blades do not bend, the rotor precone is not affected the way it is for the other codes. The other cases we ran for this condition provided no new insight.

All the models with flexible blades gave good agreement for the first fundamental modes. The first flap mode was almost $2.5 \mathrm{~Hz}$ for all codes, but there was a $2 \%$ variation in the first edge mode, which was around $7 \mathrm{~Hz}$.

\section{Teetering Turbine with Flexible Blades}

The teetering turbine with flexible blades represents the most complex configuration we used for this phase of the study. In addition to the cases we did for the simpler configurations, we ran four IEC extreme-load conditions and three 10-minute, full-field turbulence simulations.

For the conditions when the turbine operated in a vacuum or in steady winds, the results were similar to those for the teetering turbine with rigid blades. Because of the lack of blade flexibility, YawDyn differed in OoP bending moment. See Figure 5 for a plot of the blade loads when operating the turbine in a vacuum.

For the IEC extreme-load cases, $B L A D E D$ differed from the others in ways similar to those reported earlier. For the extreme-coherent-gust event, all codes gave virtually the same answers. For teeter deflection, $B L A D E D$ differed in the three cases that included a wind-direction change for this fixed-yaw turbine. YawDyn was closer to ADAMS and FAST_AD, which gave essentially identical results.

For the OoP loads, the codes gave good agreement for the EDC_r case. For the higher wind-speed cases (EDC_o and ECD), ADAMS and FAST_AD agreed perfectly, but the other codes differed by quite a bit. See Figure 6 for plots for the ECD case.

Although $B L A D E D$ has an extra peak that the AeroDyn-based codes do not have, all the codes are in good agreement for the IP loads. See Figure 7 for a plot of ECD case.

For yaw moments, BLADED differed the most, as seen in Figure 8. For the three cases with yawed flow, $B L A D E D$ 's peak-to-peak magnitudes were greater than those of the other codes. Again, ADAMS and FAST_AD gave virtually identical results. 
We next ran 10-minute simulations with full-field turbulent winds. The simulations used mean u-component wind speeds of 6,12 and $18 \mathrm{~m} / \mathrm{s}$. We analyzed the data by computing PDs, PSDs, and RCCs. In general, the agreement between ADAMS and FAST_AD was nearly perfect.

An examination of the PSDs provided few insights. For the teeter deflection, we found that $B L A D E D$ seems to have more power up to about 3P (thrice per revolution) than the other codes at $6 \mathrm{~m} / \mathrm{s}$. Differences at higher mean wind speeds were small. This seems to agree with the fact that $B L A D E D$ generally had larger teeter excursions-especially at lower wind speeds where the blade motion has a greater impact.

Examining the PDs for the teeter deflection showed the AeroDyn-based codes agreed extremely well, while the $B L A D E D$ results differed from the other codes in a way that depended on wind speed. Although $B L A D E D$ had a wider and shorter distribution, it was closer to the other codes at the higher wind speeds. See Figure 9 for the $12 \mathrm{~m} / \mathrm{s}$ case.

The OoP results showed that YawDyn differed from the other codes by a fair amount because of the rigid blades. BLADED differed by a lesser amount, but the difference increased with wind speed. Again, ADAMS and FAST_AD were quite close. For IP loads, all codes agreed quite well. For yaw moment, the AeroDynbased codes were closest, although YawDyn differed by a modest amount at $6 \mathrm{~m} / \mathrm{s}$. At $6 \mathrm{~m} / \mathrm{s}$, the AeroDynbased codes had a bimodal distribution, which indicated that the data were fairly sinusoidal. BLADED had a much weaker left hump. At the other wind speeds, all codes gave good agreement. See Figure 10 for plot of the $12 \mathrm{~m} / \mathrm{s}$ case.

Comparing the RCCs showed results similar to the other ways of analyzing the data. Once again, ADAMS and FAST_AD were most alike. Lack of flexibility in YawDyn's blades was quite apparent in the OoP dataespecially at the high wind speeds where flexibility has the greatest impact. See Figure 11 for the $18 \mathrm{~m} / \mathrm{s}$ case. Differences with BLADED's teeter deflections (not shown) and yaw moments (Figure 12) showed quite well in these plots.

\section{CONCLUSIONS}

Although we still see differences between the codes, we are getting much better agreement now than when we started this study. The many errors that were fixed certainly helped this. Careful use of only those features common to all codes and the elimination of modeling errors also improved matters. Making temporary modifications to AeroDyn to gain better agreement with $B L A D E D$ had a major impact on the comparisons.
We are quite happy with the comparisons of the blade loads. Although the predictions varied somewhat, we believe these differences are not significant when compared to the difficulty of obtaining the accurate structural and aerodynamic properties required to build the models.

We are not as comfortable with the differences in the aerodynamics, teeter motion, and yaw moments. We suspect that most of the differences in the predictions of the codes are caused by differences in the aerodynamic calculations. Therefore, in a way, this is a two-way comparison between BLADED and AeroDyn.

We need to come to a consensus on the correct induction-factor equations. The differences in predictions between $B L A D E D$ and the AeroDyn-based codes would be much greater if we had used the regular version of AeroDyn. We hope that an upcoming wind-tunnel test of a $10 \mathrm{~m}$ turbine will provide that consensus.

We do not know why BLADED has a more active teeter response than the AeroDyn-based codes. The balance between the teeter deflection and the OoP moments seems different than for the other codes. We suspect it is because there is not as much aerodynamic damping resulting from blade and/or teeter motion.

We eliminated the yaw DOF from this study because of the large differences in responses between all the codes. After abandoning free yaw, we found errors in FAST_AD and YawDyn and also made significant, albeit temporary, changes to AeroDyn, to obtain better agreement.

One benefit of the code runoff is that it provided a fairly thorough verification of our newest code, FAST_AD. Once the errors were fixed by R. Wilson, the agreement between FAST_AD and ADAMS was surprisingly good. The predictions from these two codes came closest in this study. Because FAST_AD is fast, easy to use, and free, we think designers should consider adding it to their design-code tool belt. Because YawDyn is much faster and even easier to use than FAST_AD, it is still useful for preliminary analyses when a simpler model is sufficient.

\section{FUTURE WORK}

For this phase of the study, we used only those modeling features that were common to most of the codes. We did not use the yaw DOF, tower flexibility, teeter stops, tower shadow, or dynamic inflow. If time and budget permit, we would like to test these features, eliminate the restriction of using common features, and create the best models we can with each of the codes. We think it would be appropriate to do a loads analysis that is typical of turbine designers attempting certification. We would like to know how differently the codes 
predict the lifetime and extreme loads for some turbine components.

We would also like to convert our models to simulate a three-bladed, upwind turbine with properties similar to the AWT-27CR. We would reduce the blade chord to keep the solidity the same. This would, in turn, necessitate modification of the mass and stiffness distributions.

\section{ACKNOWLEDGEMENTS}

We would like to thank everyone who helped us with this study: Ervin Bossanyi, David Quarton, and Robert Rawlinson-Smith of Garrad Hassan, who helped us use their program and taught us some of the finer details of modeling wind turbines; Craig Hansen, Dave Laino, and Akihiro Suzuki of Windward Engineering, who created YawDyn and AeroDyn, for fixing the problems we found, and helping us interface AeroDyn with the original FAST code from Oregon State University; Bob Wilson from Oregon State, who created FAST, for fixing its problems in a timely fashion and teaching us about blade-element momentum theory; and Jim Tangler of NREL for helping us with aerodynamic theory.

We would also like to thank all the managers at DOE and NREL who waited patiently over the years for us to do this study the right way. Without the extra time, we would not have been able to examine the codes with the meticulous detail needed to uncover all the errors we found. And a special thanks to Robert Thresher, Sue Hock, Mike Robinson, and Sandy Butterfield, without whom these codes would not be as good as they are today.

This work was done at the National Renewable Energy Laboratory in support of the U.S. Department of Energy under contract number DC-AC36-98-GO10337.

\section{REFERENCES}

1 Buhl, M.L., Jr.; Wright, A.D.; Tangler, J.L. Wind Turbine Design Codes: A Preliminary Comparison of the Aerodynamics. NREL/CP-500-23975. Golden, Colorado: National Renewable Energy Laboratory, December 1997.

2 Bossanyi, E.A. BLADED for Windows Theory Manual. Bristol, England: Garrad Hassan and Partners Limited, September 1997.

3 Wilson, R.E.; Walker, S.N.; Heh, P. Technical and User's Manual for the FAST_AD Advanced Dynamics Code. OSU/NREL Report 99-01. Corvallis, Oregon: Oregon State University, May 1999.
4 Hansen, A.C. User's Guide to the Wind Turbine Dynamics Computer Programs YawDyn and AeroDyn for ADAMS®, Version 11.0. Salt Lake City, Utah: University of Utah, August 1998. Prepared for the National Renewable Energy Laboratory under Subcontract No. XAF-4-14076-02.

5 International Electrotechnical Commission (TC88). Wind Turbine Generator Systems - Part 1: Safety Requirements. Second Edition. IEC 61400-1. Geneva: International Electrotechnical Commission, February 1999.

6 Kelley, N.D. Full Vector (3-D) Inflow Simulation in Natural and Wind Farm Environments Using an Expanded Version of the SNLWIND (Veers') Turbulence Code. NREL/TP-442-5225. Golden, Colorado: National Renewable Energy Laboratory, 1992.

7 Buhl, M.L., Jr.; Weaver, N.L. GPP Version 6 User's Guide. NREL/TP-500-25815. Golden, Colorado: National Renewable Energy Laboratory, July 1999.

8 Buhl, M.L., Jr. NWTC Design Codes (Crunch), 1999. http://wind2.nrel.gov/designcodes/crunch. Last modified June 11, 1999; accessed June 18, 1999.

9 Wilson, R.E.; Patton, E.M. Design Analysis of Performance and Aerodynamic Loading of NonFlexible Horizontal Axis Wind Turbines. OSU/DOE Report RLO/2227-78-2. Corvallis, Oregon: Oregon State University, August 1978; p. 68 . 


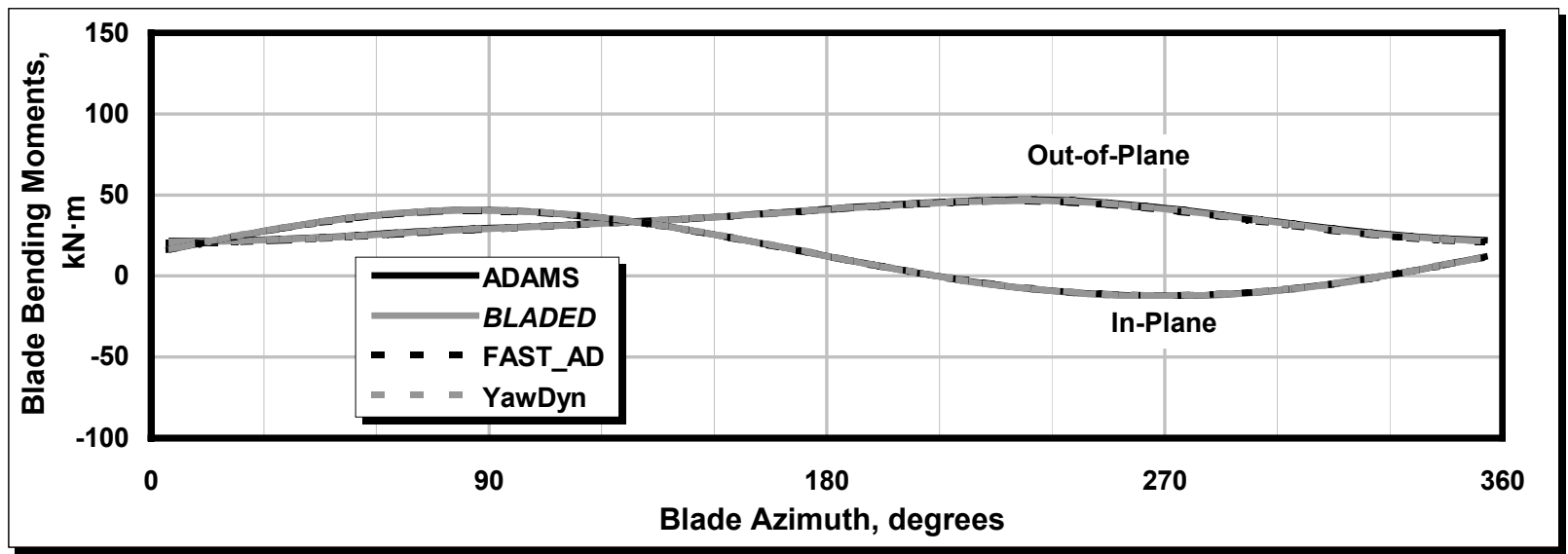

Figure 1. A comparison of blade loads for a rigid, non-teetering turbine operating with a yaw error of $30^{\circ}$ in $12 \mathrm{~m} / \mathrm{s}$, sheared, steady winds.

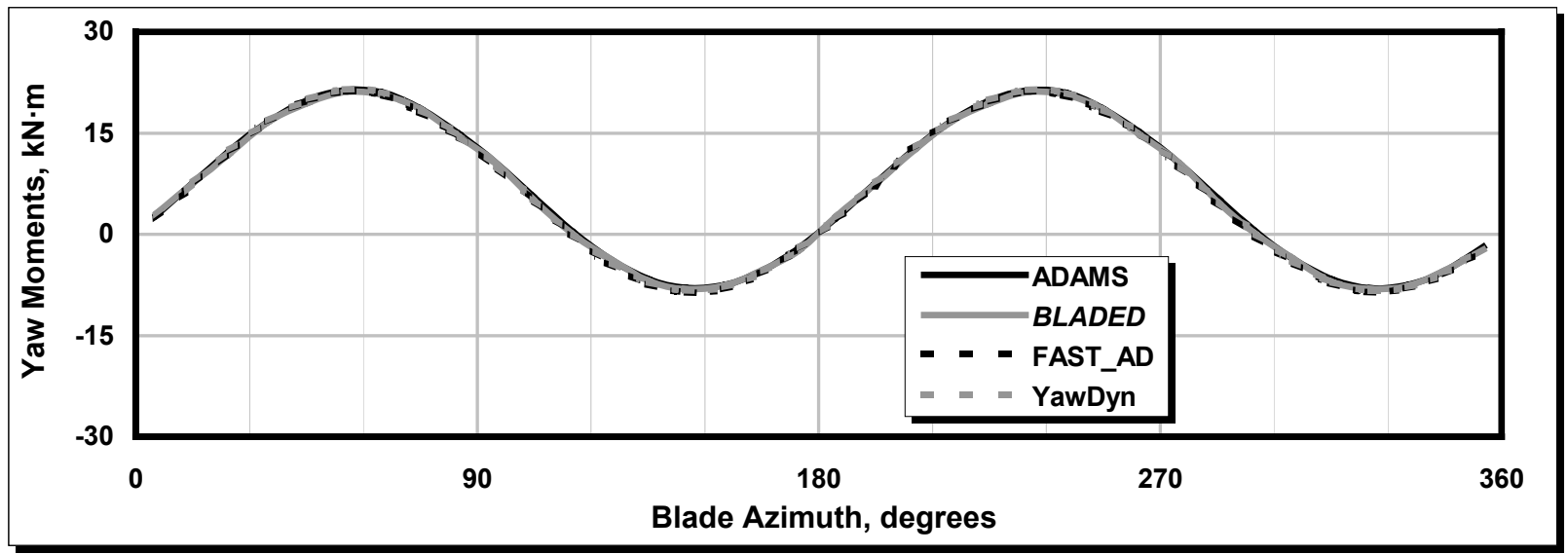

Figure 2. A comparison of yaw moments for a rigid, non-teetering turbine operating with a yaw error of $30^{\circ}$ in $12 \mathrm{~m} / \mathrm{s}$, sheared, steady winds.

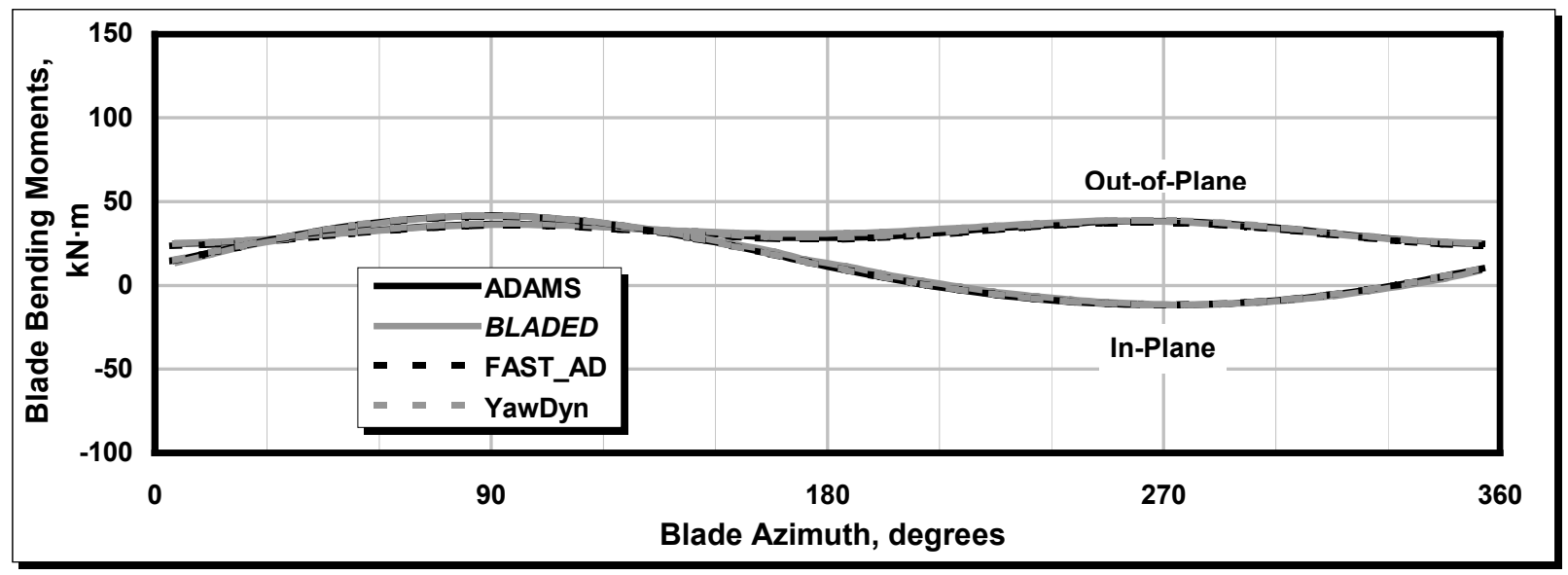

Figure 3. A comparison of blade bending moments for a teetering turbine with rigid blades operating with a yaw error of $30^{\circ}$ in $12 \mathrm{~m} / \mathrm{s}$, non-sheared, steady winds. 


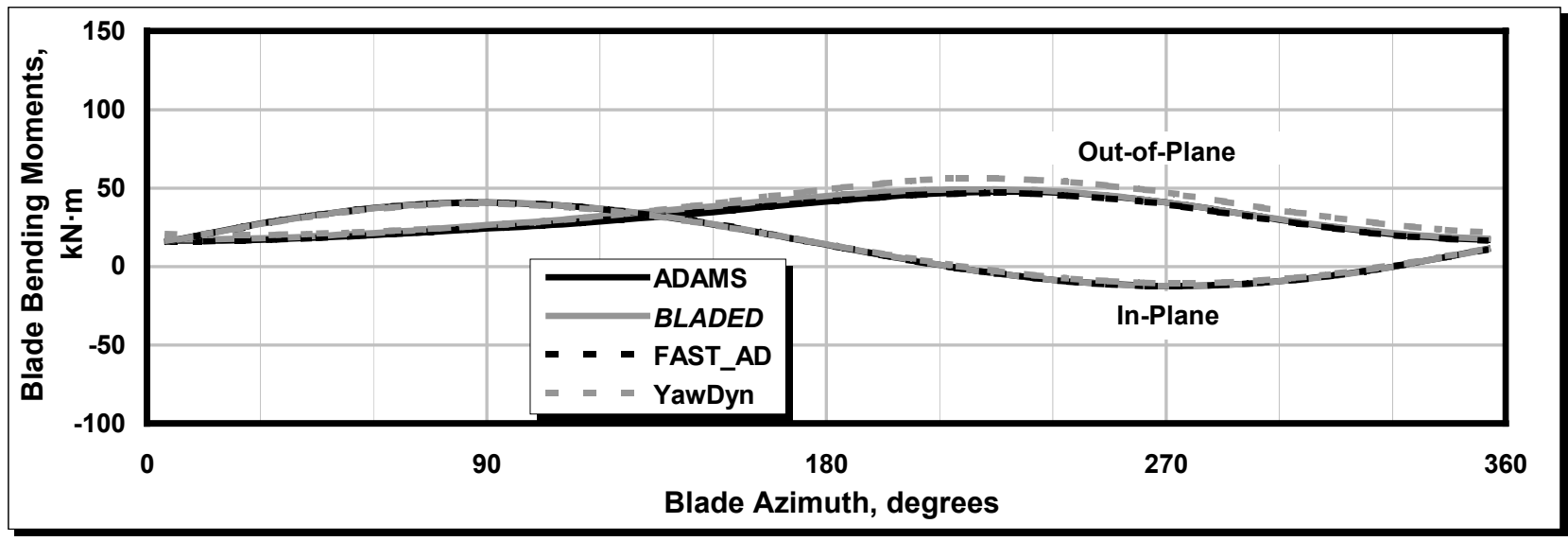

Figure 4. A comparison of blade bending moments for a non-teetering turbine with flexible blades operating with a yaw error of $30^{\circ}$ in $12 \mathrm{~m} / \mathrm{s}$, non-sheared, steady winds.

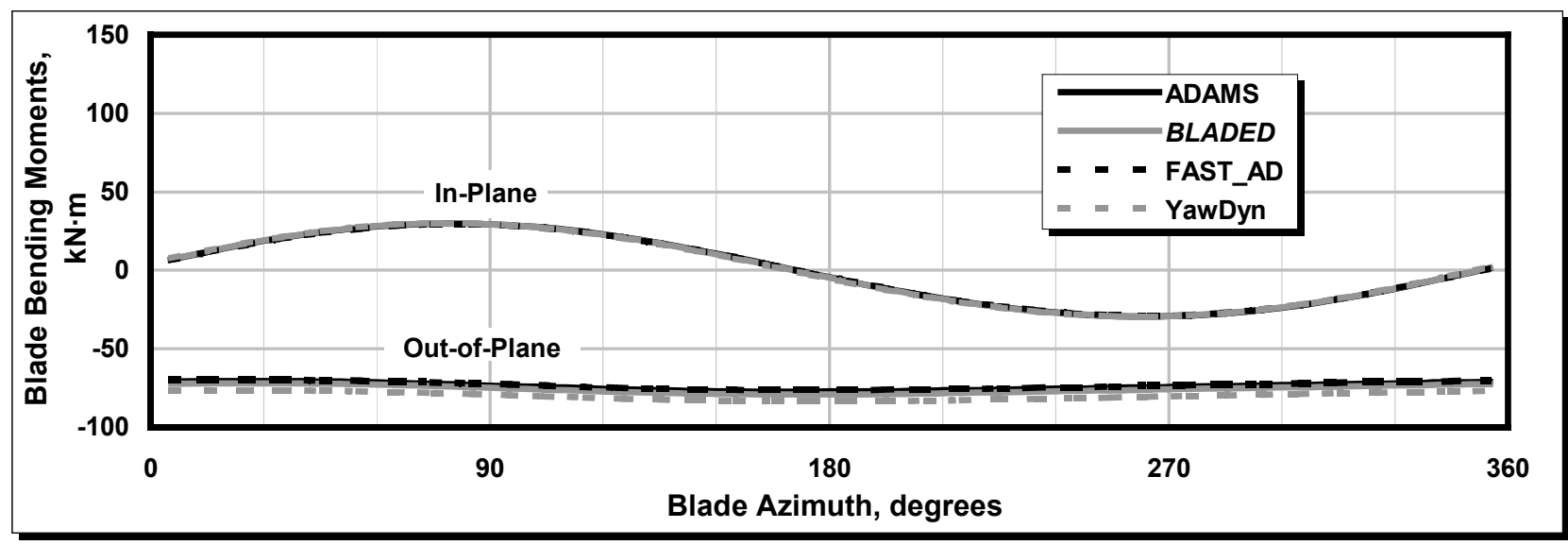

Figure 5. A comparison of blade bending moments for a teetering turbine with flexible blades operating in a vacuum.

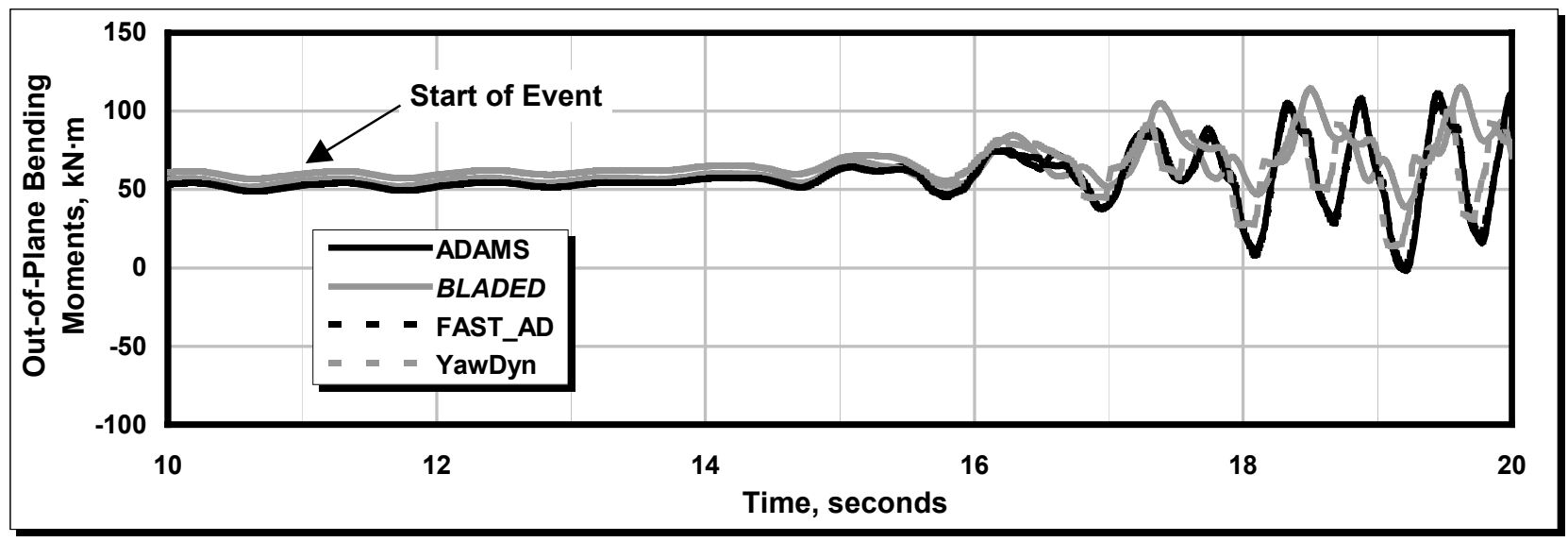

Figure 6. A comparison of blade OoP bending moments for a teetering turbine with flexible blades operating during an extreme coherent gust with direction change. 


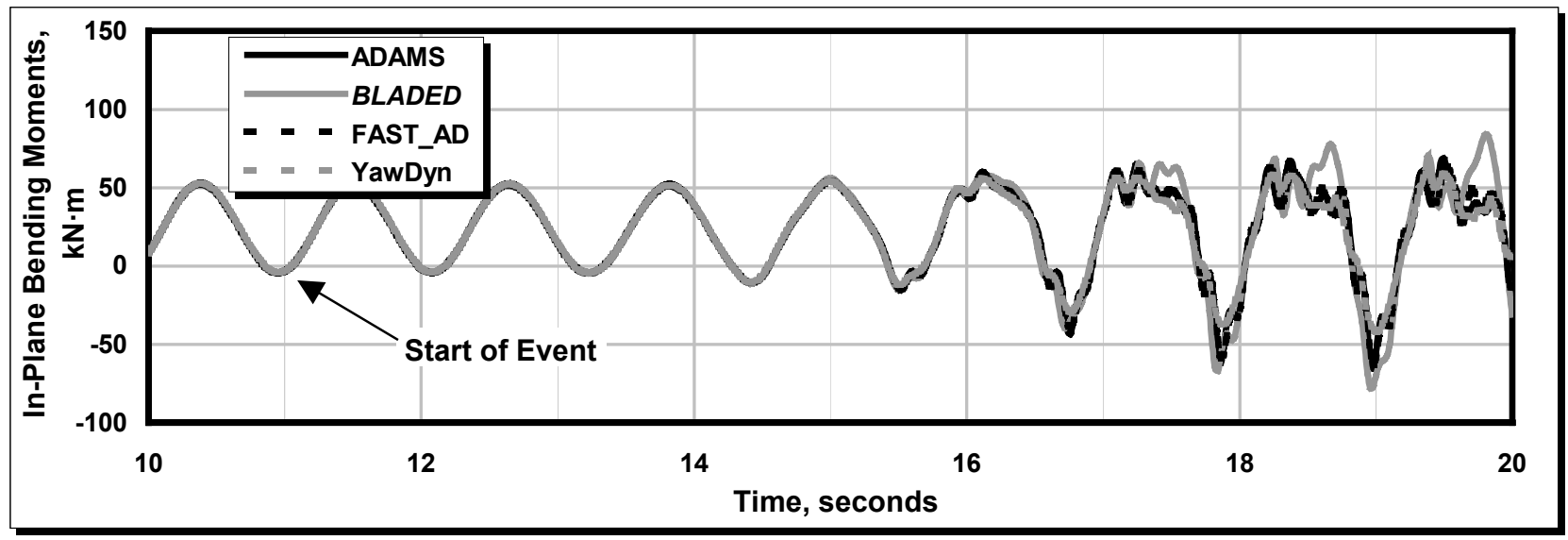

Figure 7. A comparison of blade IP bending moments for a teetering turbine with flexible blades operating during an extreme coherent gust with direction change.

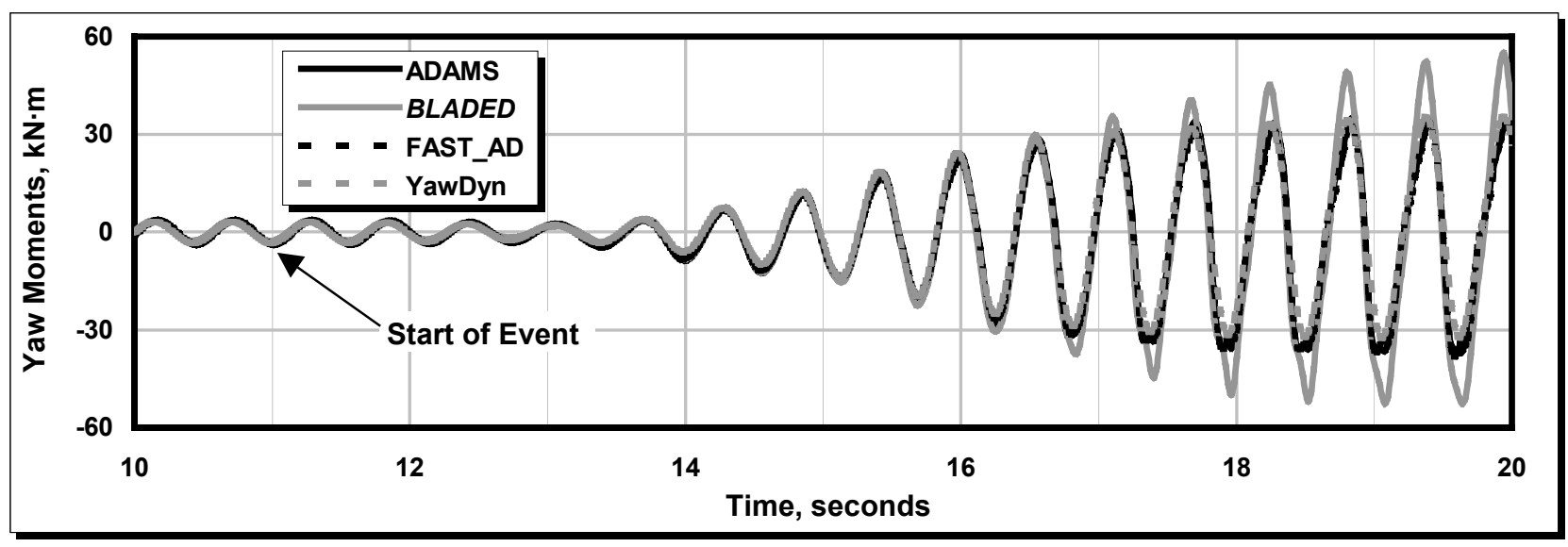

Figure 8. A comparison of yaw moments for a teetering turbine with flexible blades operating during an extreme coherent gust with direction change.

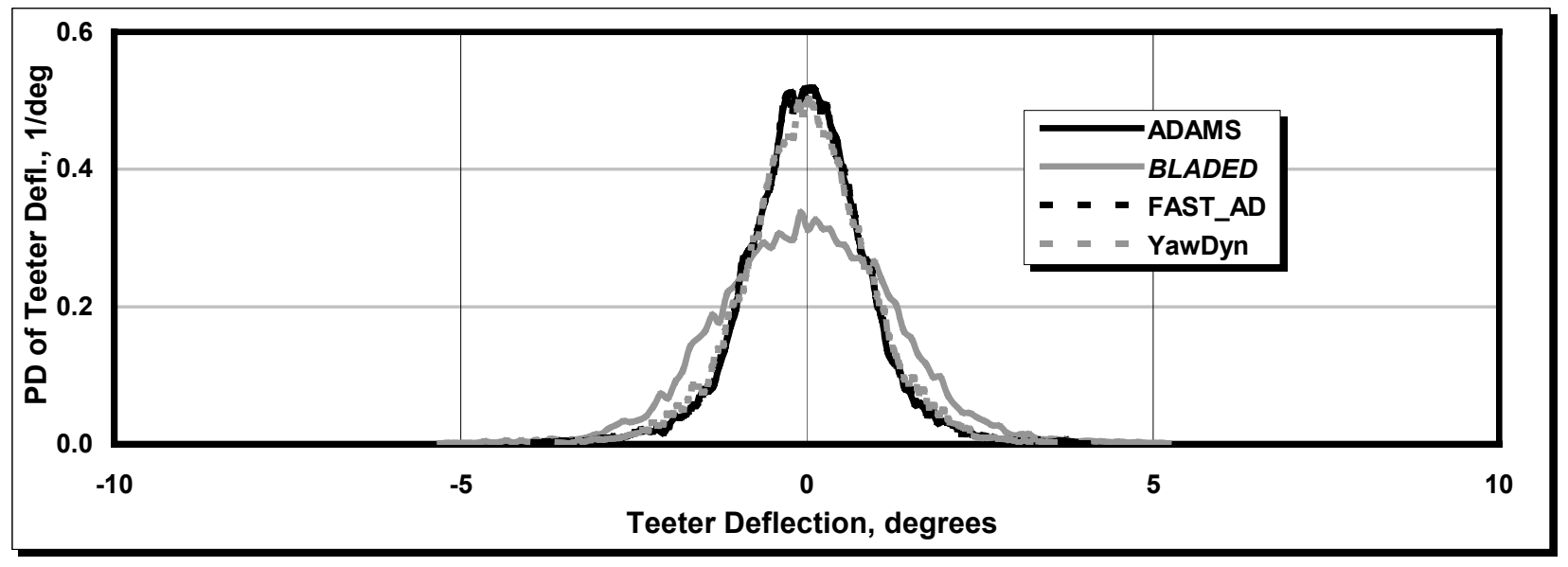

Figure 9. A comparison of PDs of teeter deflections for a $12 \mathrm{~m} / \mathrm{s}$ turbulent wind. 


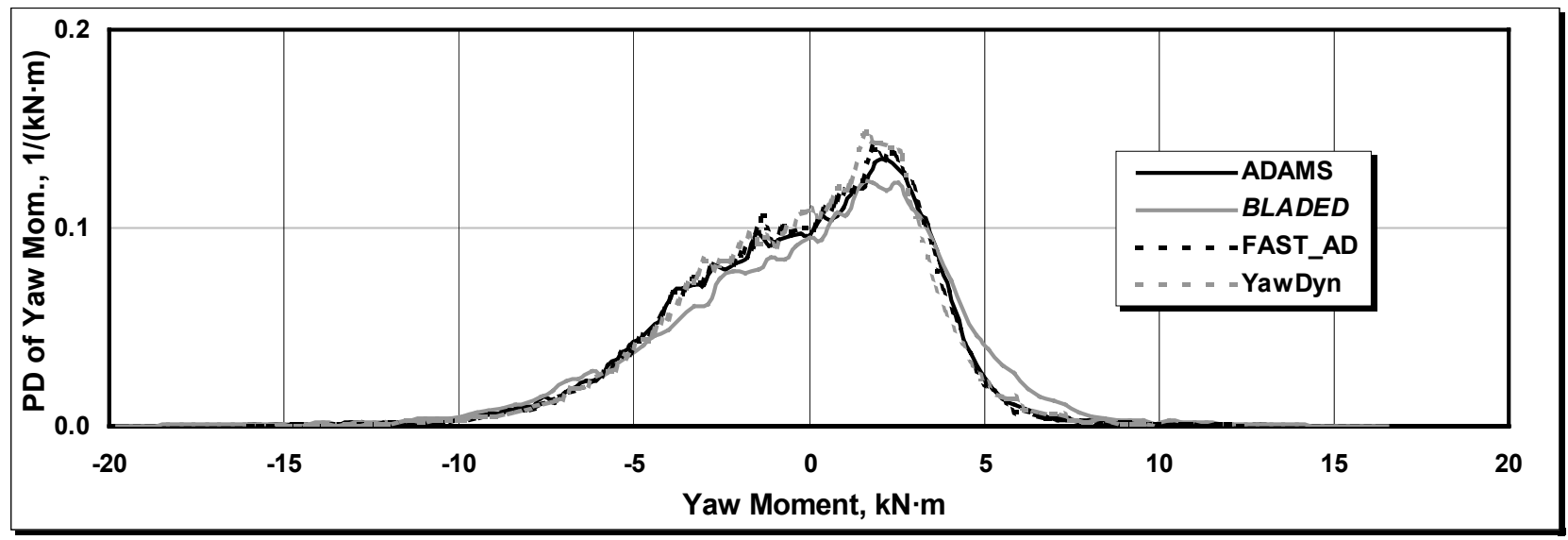

Figure 10. A comparison of PDs of yaw moments for a $12 \mathrm{~m} / \mathrm{s}$ turbulent wind.

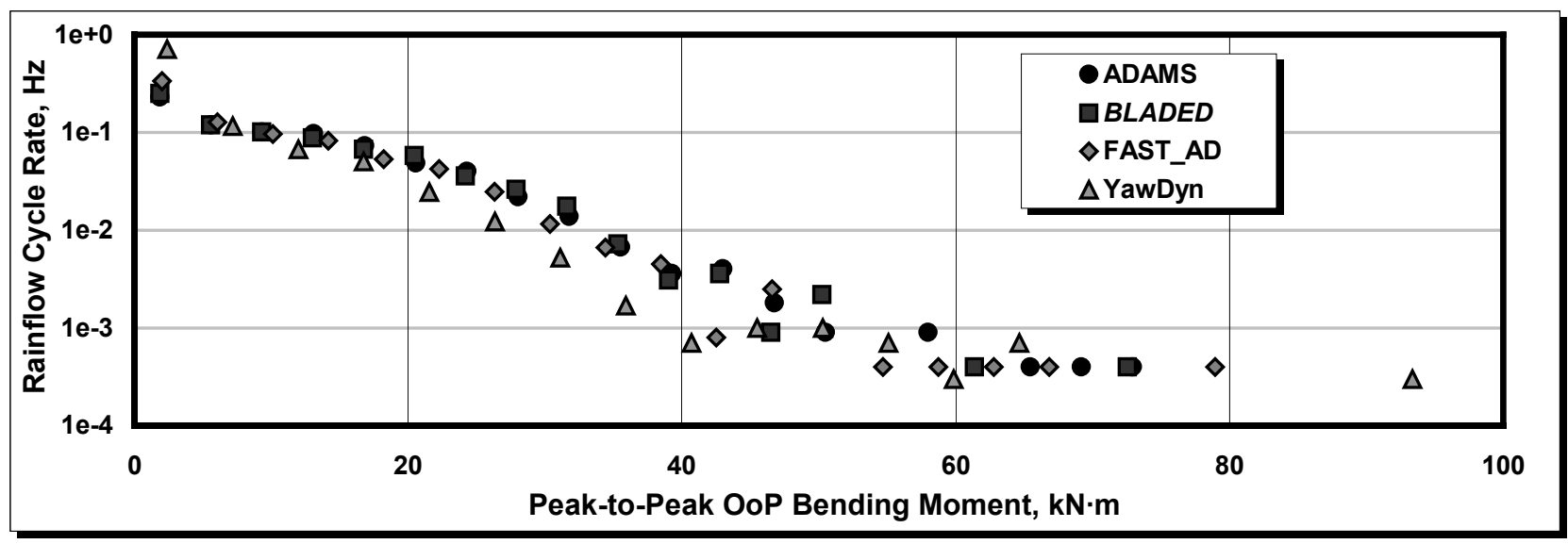

Figure 11. A comparison of Normalized RCCs of OoP bending moments for a $18 \mathrm{~m} / \mathrm{s}$ turbulent wind.

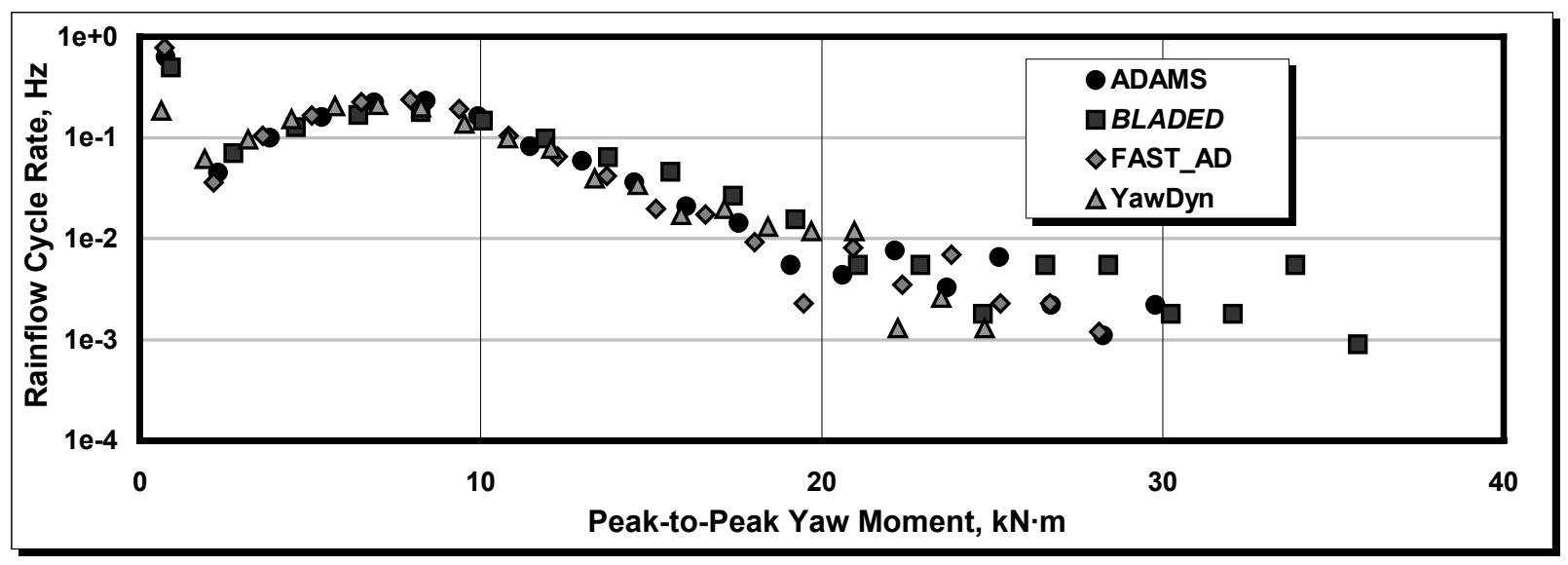

Figure 12. A comparison of Normalized RCCs of yaw moments for a $12 \mathrm{~m} / \mathrm{s}$ turbulent wind. 